Krzysztof Kotuła

Université Marie Curie-Skłodowska, Lublin

\title{
REFLECHIR SUR LES TICE EN CLASSE D'ANGLAIS: CAS DES ENSEIGNANTS EN FORMATION INITIALE
}

\author{
New technologies in the classroom: \\ The case of prospective language teachers
}

\begin{abstract}
The goal of the present paper is to question the validity of the claim which sees reflection as an important part of the work of an efficient language teacher. A quasi-experiment was conducted by the author in order to determine whether reflection is a factor which can in some way influence the teacher's capacity to plan a FL lesson effectively, incorporating new technologies. A reflective thinking questionnaire (RTQ, Kember et al., 2000) was administered to assess the level of reflective thinking in 47 university students. Next, their lesson projects were evaluated by two independent experts. The correlational study seems to show that reflection may indeed be a factor which influences the quality of the work of a foreign language teacher in an important way.
\end{abstract}

Keywords: reflective practice, CALL, ludic strategies, training prospective foreign language teachers, designing foreign language lessons

Słowa kluczowe: refleksyjna praktyka, TIK, strategie ludyczne, kształcenie przyszłych nauczycieli języka obcego, planowanie lekcji języka obcego

\section{Introduction : la notion de réflexion dans la didactique des langues étrangères}

Comme l'a fait remarquer Zawadzka, les premiers articles concernant le problème de la réflexion dans la didactique des langues étrangères apparaissent dans la littérature spécialisée à partir des années $60 \mathrm{du} \mathrm{XX}^{\mathrm{e}}$ 
siècle (2004: 299). Certes, la notion de réflexion dans les écrits pédagogiques se fait voir bien avant, il suffit de citer Dewey, qui souligne que celle-ci « nous permet d'agir d'une manière délibérée et intentionnelle [et] de savoir où nous en sommes lorsque nous agissons " ${ }^{1}$. II s'agit donc d'une forme d'activité nous donnant la possibilité de nous libérer de la routine (Farrell, 2012: 11). Actuellement, l'idée de réflexion est le plus souvent discutée en relation avec la conception de la pratique réflexive présentée par Schön dans son livre Praticien réflexif publié en 1983. Comme le souligne Schön, les praticiens - groupe dans lequel il faut certainement inclure les enseignants de langues étrangères - doivent souvent faire face à des problèmes, par nature, exceptionnels et uniques. Les personnes dotées de la capacité de réflexion peuvent se débrouiller dans des situations d'instabilité et d'insécurité. La réflexion, qui peut être comprise comme un " processus intellectuel qui consiste en la structuration ou la restructuration de l'expérience, du savoir que l'on possède, ou même de l'intuition $\|^{2}$ est donc une condition sine qua non du succès.

Schön distingue deux types de réflexion: "réflexion dans l'action" (ang. reflection-in-action) qui prend place simultanément à l'action entreprise, lorsqu'un praticien fait face à un événement qui ne se déroule pas selon ses attentes (Kinsella et al., 2012: 212), et " réflexion sur l'action » (ang. reflection-on-action) qui implique une certaine distance temporelle, lorsqu'un praticien regarde en arrière pour analyser ses actions et comportements (ibidem : 213). À ces deux catégories certains chercheurs en ajoutent encore une troisième, décrivant la réflexion qui a lieu avant l'action entreprise. Ce dernier type ne possède pas une appellation unique, les chercheurs l'appelant soit anticipatory reflection (van Manen 1991), reflection before action (Greenwood, 1993) ou bien reflection-for-action (Ghaye, 2011). Van Manen (1991 : 101) décrit ce type de réflexion comme permettant de délibérer sur les alternatives possibles, décider du cours de l'action, planifier les actions que nous devons entreprendre et anticiper les conséquences que nous et les autres pouvons subir en résultat des événements anticipés ou actions planifiées $^{3}$. II faut cependant noter qu'une partie des chercheurs rejettent

\footnotetext{
${ }^{1}$ « Act in deliberate and intentional fashion, to know what we are about when we act. » (Dewey, 1933 : 17, notre traduction).

${ }^{2}$ « Proces intelektualny, polegający na strukturyzacji bądź restrukturyzacji doświadczenia, posiadanej wiedzy, czy nawet intuicji » (Kwiatkowska 1997: 142, notre traduction). ${ }^{3}$ « Anticipatory reflection enables us to deliberate about possible alternatives, decide on courses of action, plan the kinds of things we need to do, and anticipate the experiences we and others may have as a result of expected events or of our planned actions. ".
} 
cette dernière catégorie en affirmant que ce type ne peut pas être considéré comme réflexion "pure ", car il est un mélange de réflexion et d'imagination (Moon, 1999: 58). Dans le présent article, nous n'admettons pas cette limitation, parce que vouloir restreindre la pensée réflexive à un cadre temporel rigide paraît appauvrir cette notion qui ne doit pas se borner à la seule délibération des actions passées. C'est justement ce dernier type de réflexion qui se trouve au cœur de la recherche présentée ci-dessous.

\section{Réflexion en tant que composante essentielle du travail des enseignants de langues}

Le problème qui sera discuté dans la suite de l'article peut être résumé de la façon suivante : existe-t-il un rapport entre le niveau de la réflexivité d'un (futur) enseignant de langue étrangère et sa capacité à planifier une leçon de cette langue ? Plus précisément, nous nous pencherons sur le problème de l'inclusion des TICE en classe de langues.

Nous pourrions nous interroger sur la raison de vouloir chercher un rapport entre la réflexivité et la capacité d'utiliser d'une manière efficace les TICE en cours de langue étrangère. II est pourtant hors de doute que les problèmes auxquels doit faire face un enseignant désirant incorporer les technologies dans son travail exigent une grande flexibilité, la capacité d'analyser ses erreurs et de formuler des hypothèses sur la manière optimale de planifier les actions futures, ainsi que la faculté de mettre constamment en question les bases sur lesquelles se fondent ses propres convictions.

Les difficultés découlent en grande partie de la nature des technologies elles-mêmes. Premièrement, le rythme de leur émergence est actuellement beaucoup plus rapide qu'autrefois. II suffit de suivre les dates d'apparition de quelques outils et logiciels qui ont trouvé une application en didactique des langues (p. ex. Hot Potatoes - 1998, Moodle - 2002, Second Life - 2003, lecteurs e-book - 2004, Twitter - 2006), pour constater que la rapidité du progrès est surprenante.

En deuxième lieu, les TICE sont beaucoup plus complexes que jadis, ce qui exige un effort particulier de la part des enseignants désirant exploiter pleinement leur potentiel. Les professeurs qui étaient en fin de carrière il y a quelques années à peine ont dû s'adapter seulement à quelques grands changements (tels que l'apparition du magnétoscope). Aujourd'hui, avec l'explosion des nouvelles technologies, il est parfois bien difficile de se retrouver dans cette nouvelle réalité qui oblige souvent à s'aventurer hors des sentiers 
battus. En même temps, se creuse inévitablement l'écart entre les recherches menées par les spécialistes en TICE et la réalité quotidienne de la classe.

Troisièmement, les nouvelles technologies se propagent beaucoup plus rapidement qu'autrefois. En résultat, elles deviennent très vite accessibles à une grande partie de la population. De plus, cette accélération ne cesse d'augmenter. Alors qu'il a fallu cinquante ans pour assurer une large diffusion de l'appareil téléphonique, sept ans seulement ont suffi pour l'internet (cf. la notion de technology adoption rate; King, 2013 : 28). Une autre caractéristique du développement technologique est sa non-linéarité. Par exemple, chaque nouvelle génération d'appareils portables possède un nombre important de nouvelles fonctionnalités, ce qui contribue aussi au développement de nouveaux modèles de travail avec les élèves (tels que p.ex. le mobile learning, cf. Beres, 2011).

Un autre problème caractéristique des TICE est le fait que les outils qu'un enseignant tient à sa disposition peuvent assez rapidement devenir périmés. Par exemple, un logiciel utilisé par le professeur peut cesser d'être développé ou bien un outil qui était jusque-là gratuit devient une solution payante. Cela crée forcément un sentiment d'instabilité, car l'enseignant ne peut pas toujours compter sur les méthodes de travail une fois établies, mais doit constamment y apporter des modifications.

Face aux défis actuels, il est nécessaire de repenser certains éléments de la formation de futurs enseignants. Ceci est particulièrement difficile dans le domaine des nouvelles technologies. Comme le constate à raison Amiri (2000), le rythme des changements est tellement rapide qu'il n'est plus possible de dresser une liste complète des outils que les professeurs de langues devraient maîtriser. De même, on aurait du mal à imaginer une classe de langue dans un demi-siècle, lorsque les étudiants d'aujourd'hui atteindront l'âge de la retraite. Par conséquent, un enseignement moderne doit reposer en grande partie sur l'inventivité et la réflexivité. Celle-ci est indispensable pour toutes les personnes qui souhaiteraient profiter à titre entier du potentiel des nouvelles technologies.

\section{Présentation de la recherche empirique}

\subsection{Objectifs de la recherche, sa construction et ses participants}

Le but de la recherche menée était de vérifier si la réflexivité est un facteur qui peut avoir une influence sur la capacité de planifier d'une manière logique et structurée une leçon de langue étrangère (l'anglais, en l'occurrence) 
permettant d'exploiter au maximum le potentiel d'un outil donné. 47 étudiants du Département de linguistique appliquée de I'Université Marie Curie-Skłodowska de Lublin ont pris part dans la recherche. Ils ont participé pendant l'année universitaire 2011/2012 et 2012/2013 au séminaire " Didactique des langues étrangères et TICE » dirigé par l'auteur du présent article. Les étudiants ont suivi 30 heures (un semestre) de cours consacré aux principaux concepts et médias ayant trouvé une application en didactique (Web 2.0, environnements virtuels d'apprentissage, mondes virtuels etc.). À la fin de l'année universitaire, les participants ont été priés de remplir un questionnaire. Les données ont été recueillies, en préservant l'anonymat, avec l'aide du logiciel Google Forms. Pour toutes les analyses statistiques c'est le logiciel Statistica version 8 qui a été utilisé.

Le questionnaire était composé de trois parties. La première partie de l'enquête contenait une série de questions portant sur la caractéristique générale de la personne examinée (sexe, âge, expérience éventuelle dans l'enseignement des langues, etc.). Dans cette partie, les participants ont été également priés d'évaluer leurs propres compétences informatiques, en répondant à une série de questions sur une échelle de Likert de cinq points où 1 correspondait au manque de compétence dans le domaine particulier, et 5 à la compétence pleinement développée. Ce formulaire était une version raccourcie et remaniée du questionnaire utilisé par Krajka (2012) dans sa recherche.

La deuxième partie contenait un outil servant à mesurer le niveau de la pensée réflexive établi par Kember et al. (2000). Ce questionnaire se compose de seize assertions, présentées en ordre aléatoire, dont chacune appartient à une des quatre catégories distinctes: actions habituelles, compréhension, réflexion et réflexion critique. Les participants sont priés d'émettre des jugements sur leurs habitudes, comportements et processus mentaux en prenant position par rapport à chacune des affirmations en jugeant de leur conformité avec la vérité sur une échelle de Likert de cinq points où 1 veut dire "je suis entièrement en désaccord" et 5 " je suis entièrement en accord ». Comme le souligne Perkowska-Klejman (2012), la réflexion est définie par Kember et ses collaborateurs comme la capacité de l'être humain à mettre en question sa façon de résoudre les problèmes: le sujet se demande si les défis auxquels il a dû faire face ont été résolus d'une manière optimale et avec l'utilisation des meilleures méthodes. De même, en se basant sur les expériences passées, un homme réflexif est capable de prévoir les sources potentielles de problèmes à l'avenir et de planifier ses actions. En théorie donc, un praticien qui obtient un score élevé dans les deux dernières catégories devrait avoir la capacité de résoudre efficacement les problèmes. C'est ce qu'ont confirmé certaines études prouvant que les 
résultats du test RTQ corrèlent avec la capacité des individus à faire face à des défis de différents types (cf. Lucas et Leng Tan, 2006 ; Huy Phan, 2006).

La troisième partie du questionnaire comportait une série de questions ouvertes concernant l'utilisation, dans le cadre d'un cours de langue anglaise, d'un jeu vidéo Pierre Hotel. C'est un jeu d'aventure graphique en pointer-etcliquer réalisé en technologie flash, racontant l'histoire d'un couple en voyage de noces qui passe la nuit dans un hôtel hanté. Ce problème particulier a été choisi pour trois raisons distinctes. Premièrement, la question de l'implémentation des stratégies vidéoludiques ${ }^{4}$ en didactique n'a pas été discutée pendant le séminaire. Il s'agissait donc d'un sujet neuf, appartenant à un domaine inexploré. En second lieu, l'usage de ce type de techniques dans la pratique quotidienne des établissements scolaires est toujours très marginal. Il était donc probable que les étudiants n'ont jamais rencontré un problème de ce genre et, par conséquent, devaient avoir recours avant tout à leur propre inventivité et réflexivité pour le résoudre. Troisièmement, il s'agit d'un défi d'une grande complexité, ce qui exigeait des étudiants de réfléchir sur un grand nombre de questions telles que le sens de l'utilisation d'un logiciel de ce type en cours de langue étrangère, le groupe d'âge potentiellement ciblé, le niveau de langue des élèves approprié pour l'utilisation de ce genre de techniques, la configuration de la classe, le choix du logiciel et du matériel, la planification des exercices et la répartition des tâches, l'organisation efficace du travail de tous les acteurs impliqués, le choix des méthodes optimales servant à évaluer le travail des étudiants, le développement des activités extra-scolaires. etc... II s'agit donc d'un problème se caractérisant par un haut degré de complexité et unique dans sa nature.

\subsection{Résultats de la première partie de la recherche - quelques données essentielles sur les participants et leurs compétences informatiques}

Parmi les étudiants qui ont rempli l'enquête, il y a avait 45 femmes et 2 hommes âgés de 21 à 23 ans. Toutes les personnes interrogées déclaraient posséder déjà une certaine expérience dans l'enseignement des langues étrangères (entre deux et trois années, $\bar{x}=2,6)$, acquise à travers des stages pédagogiques, cours particuliers ou travail dans des écoles de langue. Il est difficile de juger de la durée réelle de leur expérience, car le seul indicateur viable dans ce cas est le nombre d'heures passées à exercer la profession. En tout cas, il s'agissait de professeurs débutants. Le groupe était donc assez homogène, ce qui est tout à

${ }^{4} \mathrm{~J}$ 'emprunte ce terme à Berry (2011). 
fait compréhensible si l'on prend en compte le fait qu'il était composé d'étudiants de troisième et quatrième année du même département.

Priés d'évaluer leurs propres compétences en tant qu'enseignants de langue étrangère (sur une échelle de 1 à 10), ils ont été plutôt positifs sur leurs capacités, la note minimale qu'ils se sont attribuée étant de 5 (une seule occurrence) et maximale de $8(\bar{x}=6,9, \sigma=0,91)$, ce qui montre qu'il sont confiants et sûrs d'eux. En ce qui concerne les questions relatives aux compétences informatiques qu'ils maîtrisent le mieux, les étudiants ont énuméré la capacité de télécharger et d'installer les logiciels, d'utiliser les éditeurs d'image ou encore les communicateurs tels que Skype. Par contre, la plupart des participants de l'enquête se sont jugés incapables d'accomplir des tâches plus complexes, telles que créer une image disque, programmer ou encore créer des sites Internet. II semble donc que la plupart des personnes interrogées ne sachent pas exploiter pleinement le potentiel des technologies Web 2.0 et utilisent les ressources électroniques d'une manière plutôt passive. L'ensemble des données est présenté sur le graphique 1.

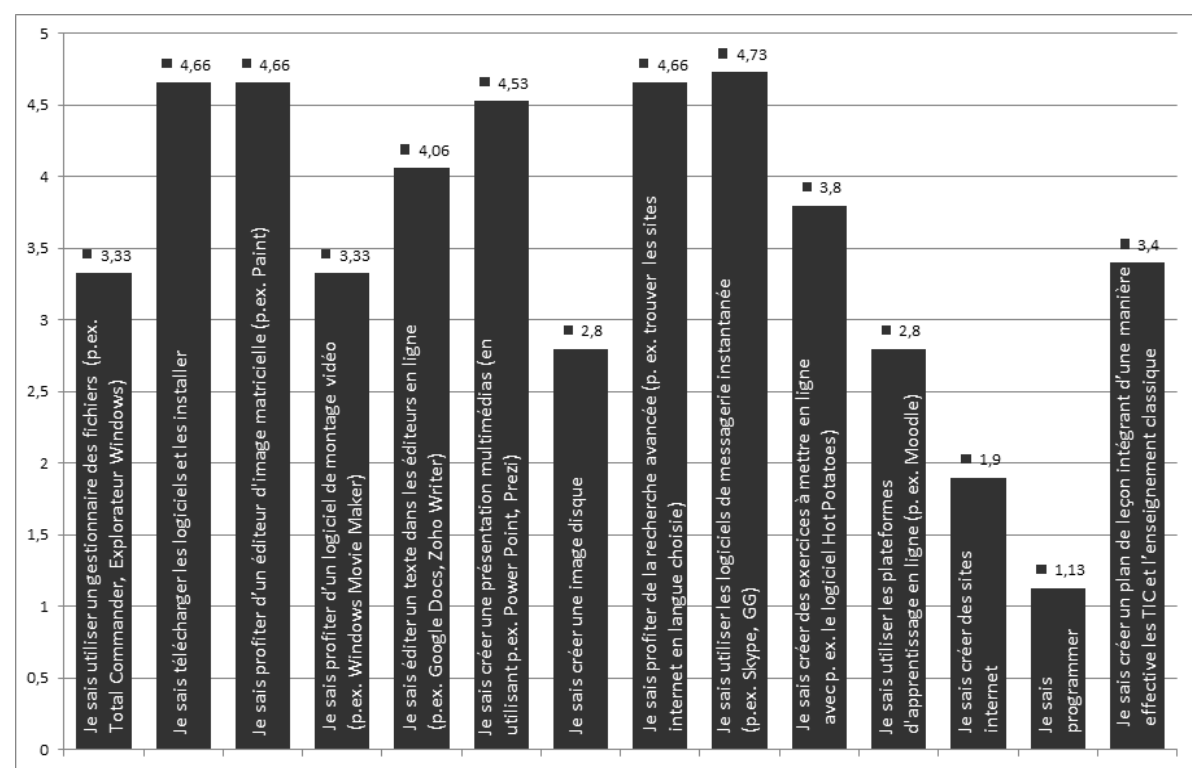

Graphique 1 : Sommaire des compétences informatiques des participants de l'enquête. Chacun des items devait être noté sur une échelle de 1 (note minimale) à 5 (note maximale). Le chiffre sur l'extrémité de chaque barre est une moyenne arithmétique pour chacune des réponses. 


\subsection{Résultats de la deuxième partie de la recherche - questionnaire RTQ}

Quant au questionnaire RTQ, en premier lieu le coefficient alpha de Cronbach a été calculé afin de mesurer la cohérence des questions appartenant à chacun des quatre groupes (actions habituelles, compréhension, réflexion et réflexion critique; cf. Kember et al., 2000). Ceci était nécessaire, car pour établir le score définitif des participants sur les quatre échelles, les valeurs numériques correspondant à chacune des réponses ont dû être additionnées. Dans le cas où les écarts entre les items seraient trop grandes, il aurait été incorrect de prendre ces données comme point de repère, ce qui remettrait en cause la validité de la recherche. Les résultats recueillis (cf. tableau 1) montrent que, dans la plupart des cas, les valeurs obtenues ont été suffisantes, c'est seulement dans le cas des actions habituelles que le score a manqué de peu d'atteindre le seuil de 0,7 , qui est généralement considéré comme acceptable. En ce qui concerne les variables " réflexion" et "réflexion critique", les valeurs du coefficient alpha de Cronbach étaient les plus élevées parmi celles qui ont été obtenues dans les quatre autres études citées dans le tableau 1.

\begin{tabular}{|l|r|r|r|r|r|}
\hline & $\begin{array}{c}\text { Kember et al. } \\
2000\end{array}$ & $\begin{array}{c}\text { Lucas et Leng } \\
\text { Tan 2006 }\end{array}$ & $\begin{array}{c}\text { Huy Phan } \\
\text { 2006 }\end{array}$ & $\begin{array}{c}\text { Perkowska- } \\
\text { Klejman 2012 }\end{array}$ & Kotuła 2013 \\
\hline Actions habituelles & 0,62 & 0,65 & 0,91 & 0,84 & 0,69 \\
\hline Compréhension & 0,76 & 0,74 & 0,79 & 0,85 & 0,82 \\
\hline Réflexion & 0,63 & 0,81 & 0,80 & 0,75 & 0,82 \\
\hline Réflexion critique & 0,67 & 0,87 & 0,85 & 0,86 & 0,93 \\
\hline
\end{tabular}

Tableau 1 : Fiabilité (coefficient $\alpha$ de Cronbach) des quatre parties du questionnaire RTQ (Kember et al. 2000). Résultats de quelques recherches choisies; la dernière colonne présente les résultats de notre recherche.

Il est également intéressant de voir comment les résultats recueillis dans la présente recherche se situent par rapport à ceux publiés antérieurement. C'est l'étude de Perkowska-Klejman (2012) qui va ici servir de point de repère : une recherche récente menée dans le contexte polonais sur un groupe de dimensions comparables. Même si les résultats des deux tests diffèrent un peu, une tendance commune peut être remarquée : les participants ont obtenu les meilleurs résultats sur la troisième échelle, alors que les autres scores y étaient inférieurs (cf. tableau 2 et graphique 2). 
Reflechir sur les tice en classe d'anglais: Cas des enseignants en formation initiale

\begin{tabular}{|l|r|r|r|r|}
\hline & \multicolumn{2}{|c|}{$\begin{array}{c}\text { Perkowska-Klejman 2012 } \\
\mathrm{N}=49\end{array}$} & \multicolumn{2}{c|}{$\begin{array}{c}\text { Kotuła 2013 } \\
\mathrm{N}=47\end{array}$} \\
\hline & $\bar{x}$ & $\sigma$ & $\bar{x}$ & $\sigma$ \\
\hline Actions habituelles & 10,08 & 3,40 & 13 & 3,08 \\
\hline Compréhension & 14,2 & 3,18 & 16 & 3,44 \\
\hline Réflexion & 17,26 & 2,08 & 17,46 & 2,44 \\
\hline Réflexion critique & 12,4 & 3,40 & 13,86 & 4,04 \\
\hline
\end{tabular}

Tableau 2: Comparaison des résultats du test RTQ employé dans la présente recherche avec ceux de Perkowska-Klejman (2012). Sont données les valeurs de la moyenne arithmétique (colonne de gauche) et celles de l'écart type (colonne de droite).

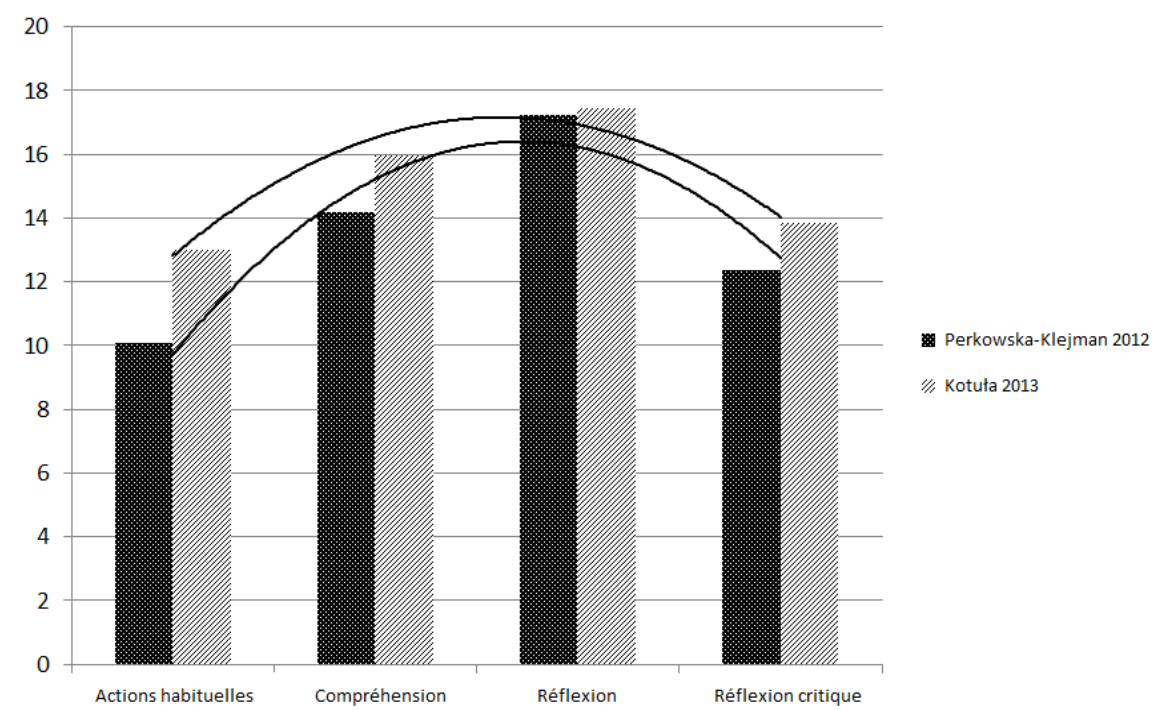

Graphique 2 : Représentation graphique des résultats moyens des participants sur chacune des quatre échelles du questionnaire RTQ dans deux recherches choisies : la nôtre et celle de Perkowska-Klejman (2012). Une courbe de tendance a été ajoutée au-dessus des barres.

\subsection{Résultats de la troisième partie de la recherche - étude corrélationnelle des variantes}

S'agissant de la troisième partie de la recherche, les réponses des participants ont été notées par l'auteur de la présente étude et un enseignant d'anglais sur une échelle de 1 à 10 . Comme les notes des deux évaluateurs n'étaient pas sensiblement différentes, une simple moyenne arithmétique a été administrée pour établir le score final. Le niveau des réponses était varié, ce qui trouve son 
reflet dans les scores : le score minimal était de 2,5, maximal de $7,83(\bar{x}=5,1$, $\sigma=1,35)$. Les réponses ont été notées suivant un protocole préétabli, prenant en compte, entre autres, la capacité de présenter un modèle de travail convaincant ainsi que de prévoir les difficultés de nature différente (organisation des activités de classe, configuration du matériel, etc.). II faut noter que les réponses qui présentaient une analyse critique de l'outil proposée (points faibles, inconsistances) ont été particulièrement appréciées.

Comme les variables obtenues dans la deuxième et troisième partie du questionnaire n'étaient pas distribuées selon la loi normale, le test nonparamétrique de Spearman a été appliqué pour trouver les corrélations. Cette analyse permet de faire quelques observations intéressantes (cf. tableau 3). Tout d'abord, le niveau de la pensée réflexive est un facteur qui a significativement corrélé avec le score des participants. La force de ce lien est modérée. Ce lien est bien plus faible dans le cas de la réflexion critique, compréhension et actions habituelles, valeurs dont seulement la première est significative. En ce qui concerne les compétences informatiques, il s'est avéré qu'elles sont également un facteur qui a eu un impact sur le score final, ce qui en principe ne devrait pas étonner vu que le sujet du test était relatif à l'emploi des nouvelles technologies dans l'enseignement des langues étrangères. Pourtant, parmi toutes les questions de la troisième partie du questionnaire, seulement une se référait directement au domaine des TICE, demandant à l'intervenant de choisir une configuration optimale du matériel.

Par contre, aucune corrélation n'a été observée entre l'auto-évaluation des compétences pédagogiques et le score obtenu. C'est une conclusion intéressante, même si le résultat d'un seul test ne peut pas suffire à déterminer si un sujet est un bon ou un mauvais enseignant. De même, aucune corrélation n'a été trouvée entre les compétences informatiques et l'auto-évaluation des compétences pédagogiques $(R=0,04 ; p=0,75)$.

\begin{tabular}{|l|r|r|r|r|r|r|}
\hline & \multicolumn{2}{|c|}{ Les quatre catégories du questionnaire RTQ } & \multicolumn{2}{c|}{ Autres variables } \\
\hline & Réflexion & $\begin{array}{c}\text { Réflexion } \\
\text { critique }\end{array}$ & Compréhension & $\begin{array}{c}\text { Actions } \\
\text { habituelles }\end{array}$ & $\begin{array}{c}\text { Auto- } \\
\text { Compétences } \\
\text { informatiques } \\
\text { des } \\
\text { dempétences } \\
\text { pédagogiques }\end{array}$ \\
\hline Score & $0,46^{* *}$ & $0,29^{*}$ & 0,14 & 0,10 & $0,41^{* *}$ & $-0,09$ \\
\hline
\end{tabular}

Tableau 3 : Valeur du coefficient de Spearman pour les corrélations avec la variable "score ». Sont marquées avec un astérisque les corrélations dont le coefficient $p$ était $<0,05$; avec un double astérisque celles dont le coefficient $p$ était $<0,01$. 
Une analyse de régression linéaire a été réalisée pour deux variables trouvées précédemment significativement corrélées avec le score des participants : "réflexion » et " compétences informatiques». Dans le cas de la variable " réflexion critique » ainsi que les autres variables pris en compte dans notre recherche, le modèle de régression s'est avéré être non-significatif. Comme nous pouvons le voir en analysant le contenu du tableau 4, le prédicteur « réflexion » explique $21 \%$ de la variance du score obtenu par les participants.

\begin{tabular}{|l|l|r|r|r|r|c|}
\hline & $\mathrm{R}^{2}$ & $\mathrm{R}^{2}$ ajusté & $\beta$ standardisé & $\mathrm{F}$ & $\mathrm{t}$ & $\mathrm{p}$ \\
\hline Réflexion & 0,21 & 0,20 & 0,46 & 12,59 & 3,54 & 0,00 \\
\hline
\end{tabular}

Tableau 4 : Analyse de régression linéaire. Variable dépendante : « score ».

Quant au prédicteur " compétences informatiques ", celui-ci explique $14 \%$ de la variance du score obtenu par les participants.

\begin{tabular}{|l|r|r|r|r|r|c|}
\hline & \multicolumn{1}{|c|}{$\mathrm{R}^{2}$} & $\mathrm{R}^{2}$ ajusté & $\beta$ standardisé & $\mathrm{F}$ & $\mathrm{t}$ & $\mathrm{p}$ \\
\hline $\begin{array}{l}\text { Compétences } \\
\text { informatiques }\end{array}$ & 0,14 & 0,12 & 0,37 & 7,48 & 2,73 & 0,00 \\
\hline
\end{tabular}

Tableau 5 : Analyse de régression linéaire. Variable dépendante : « score ».

En somme, il est visible que même si nous avons réussi à identifier deux facteurs qui ont influencé à un certain degré le score des participants, d'autres éléments encore, que nous n'avons pas pris en compte dans la présente recherche, jouent ici un rôle important. La question mérite sans doute une investigation plus approfondie.

\section{Conclusions}

II en résulte de ce qui précède que la réflexivité peut en effet être un des facteurs ayant une influence sur la capacité des futurs enseignants de planifier les activités de classe demandant une utilisation active des TICE. Cette observation a pourtant besoin d'être approfondie. S'il est vrai que la réflexion est une composante tellement importante du métier de professeur de langues, cela nous amène à la question suivante : pouvons-nous la développer et surtout comment? Le séminaire dirigé par l'auteur du présent article se donnait comme objectif non seulement de faire connaître à l'auditoire les TICE pouvant être utiles en classe, mais aussi de créer chez les étudiants l'habitude de réfléchir sur les actions passées et futures, d'analyser leurs points forts et faibles, de savoir identifier les sources d'un échec potentiel. Chaque fois qu'une nouvelle technologie était discutée, les étudiants étaient priés de 
préparer des simulations de leçons où une partie du groupe jouait le rôle des élèves. Ceci permettait d'observer les technologies en action et de prendre conscience de la complexité et de la dynamique d'une classe de langues. Reste à savoir dans quelle mesure ce type de procédé peut être efficace.

Quant aux limites de la présente étude, elles sont de triple nature. Premièrement, en se limitant aux étudiants d'un seul département ayant fréquenté le même séminaire à un an d'écart à peine, de nombreux facteurs qui pourraient avoir un impact important sur les résultats tels que p.ex. âge, éducation ou encore la longueur de l'expérience professionnelle, n'ont pas été pris en compte. Une recherche menée sur un groupe plus nombreux et diversifié aurait pu donner une image plus complète du problème. En second lieu, la capacité de planifier les leçons est certainement essentielle pour un professeur de langue étrangère, mais également importante est l'exécution, c'est-à-dire la capacité de mettre en œuvre les plans pédagogiques. II serait intéressant de voir si et dans quelle mesure la réflexivité influence la qualité du travail réel des enseignants. Troisièmement, il serait intéressant d'analyser - par exemple dans le cadre d'une étude longitudinale - le réflexivité dans une perspective plus large, non pas comme un attribut immuable d'un individu mais plutôt comme une caractéristique qui peut évoluer avec le temps. Les recherches futures vont, sans doute, apporter d'intéressantes réponses à ces questions.

\section{BIBLIOGRAPHIE}

Amiri, F. 2000. " IT-literacy for language teachers: should it include computer programming? ". System, No. $28: 77-84$.

Beres, D. L. 2011. « Mobile-assisted language learning from the student perspective: encouraging effective language learning strategies outside of the classroom » (in:) Academic podcasting and mobile assisted language learning: applications and outcomes (éd. B. R. Facer et M. Abdous). Hershey : ISR : 93-110.

Berry, V. 2011. "Jouer pour apprendre : est-ce bien sérieux ? Réflexions théoriques sur les relations entre jeu (vidéo) et apprentissage ». Canadian Journal of Learning and Technology, No. 37 : 1-14.

Dewey, J. 1933. How we Think. New York : Prometheus Books.

Farrell, T. 2012. "Reflecting on reflective rractice : (Re)Visiting Dewey and Schön ». TESOL Journal No. 3 : 7-16.

Ghaye, T. 2011. Teaching and Learning Through Reflective Practice: A Practical Guide for Positive Action. London : David Fulton Press.

Greenwood, A. 1993. "Reflective practice: a critique of the work of Argyris and Schön ». Journal of Advanced Nursing, No. 22 : 226-234. 
Huy Phan, P. 2006. « Examination of student learning approaches, reflective thinking, and epistemological beliefs: A latent variables approach ». Electronic Journal of Research in Educational Psychology, No 10: 577-610.

Kember, D., D. Leung, A. Jones et A. Y. Loke. 2000. « Development of a questionnaire to measure the level of reflective thinking ". Assessment and Evaluation in Higher Education No. 25 : 381-395.

King, B. 2013. Bank 3.0: Why Banking is no Longer Somewhere you Go but Something you Do. New York: Marshall Cavendish.

Kinsella E. A., M.-E. Caty, S. Ng et K. Jenkins. 2012. "Reflective Practice for Allied Health : Theory and Applications " (in:) Adult Education and Health (éd. L. H. English). Toronto : University of Toronto Press : 210-228.

Krajka, J. 2012. The Language Teacher in the Digital Age - Towards a Systematic Approach to Digital Teacher Development. Lublin : Wydawnictwo UMCS.

Kwiatkowska, H. 1997. Edukacja nauczycieli: konteksty - kategorie - praktyki. Warszawa : Instytut Badań Edukacyjnych.

Lucas, U. et P. Leng Tan. 2006. " Assessing levels of reflective thinking: the evaluation of an instrument for use within accounting and business education ". Présentation lors du colloque : Pedagogic Research in Higher Education Conference. Liverpool Hope University, Liverpool.

Moon, J. A. 1999. Reflection in Learning and Professional Development : Theory and Practice. New York : Kogan Page Limited.

Perkowska-Klejman, A. 2012. "Czy twoi studenci są refleksyjni? ». Studia Edukacyjne, No. $21: 211-232$.

Schön, D. A. 1983. The Reflective Practitioner: How Professionals Think in Action. New York : Basic Books.

van Manen, M. 1991. The Tact of Teaching: The Meaning of Pedagogical Thoughtfulness. New York : State University of New York Press.

Zawadzka, E. 2004. Nauczyciele języków obcych w dobie przemian. Kraków : Impuls. 